\title{
Frequent Allelic Loss and Reduced mRNA Expression of ING4, A Novel Candidate Tumor Suppressor Gene, in Head and Neck Cancer
}

\author{
Mehmet Gunduz'), Esra Gunduz¹), Wan H. W. Harun²), Fathillah Abdul Razak²), Phaik Leng Cheah'), Kenji \\ Shimizu ${ }^{1)}$, Kazunori Nishiyama ${ }^{3)}$, Noriyuki Nagai ${ }^{1)}$ \\ ${ }^{1}$ Graduate School of Medicine, Dentistry and Pharmaceutical Sciences Okayama University, Okayama, Japan \\ ${ }^{2}$ Department of Oral Biology, University of Malaya, Malaysia \\ ${ }^{3}$ Japan Institute for Advanced Dentistry, Osaka
}

\begin{abstract}
We previously showed two members of the ING family, ING1 and ING3 as a tumor suppressor gene in head and neck cancer. A novel member of ING family, ING4 localized to chromosome 12p13.31 region was cloned. ING4 harbors the PHD domain highly homologous among ING family proteins. Loss of heterozygosity analysis was performed at 12p12-13 region in 50 head and neck squamous cell carcinomas by using six highly polymorphic microsatellite markers and 33 out of the 50 cases (66\%) showed allelic loss. Although somatic mutation of the ING4 gene was not found in head and neck cancers, quantitative real-time RT-PCR analysis demonstrated decreased expression of ING4 mRNA in 76\% of primary tumors as compared with that of matched normal samples. Frequent deletion and decreased mRNA expression of ING4 suggested it as a class II tumor suppressor gene and may play an important role in head and neck cancer.
\end{abstract}

Key words: LOH, ING family, ING1, HNSCC

\section{Introduction}

A new class of tumor suppressor genes (TSGs), with haploid insufficiency, in which one allele is lost and the remaining allele is haploinsufficient, has been described recently, and these hemizygous tumor suppressor genes show a tumor-prone phenotype when challenged with carcinogens ${ }^{1}$.

One of the critical steps for the identification of TSGs is loss of heterozygosity analysis (LOH). By this method, we previously showed ING1 and ING3 as a class 2 TSGs in head and neck squamous cell carcinomas (HNSCC) ${ }^{2,3)}$. Both ING1 and ING3, which belong to ING family genes are rarely mutated but reveal a high allelic deletion rate. Chromosome 12p12-13 region has also known to be deleted in various cancers including hematological malignancies, breast cancer, prostate cancer, non-small cell lung cancer and nasopharyngeal carcinomas. Since it's been shown some of the ING family members functioning as TSG, it is likely that other members of this family could be a TSG. ING4, a new member of ING family, was localized to this frequently deleted region of $12 \mathrm{p} 13.31$.

In the current study, we examined the ING4 locus for allelic deletion in HNSCC. We also analyzed mutation and mRNA expression level of ING4 in HNSCC.

Materials and methods
Tissue samples
Paired normal and tumor samples were obtained from 50
patients with primary HNSCC at the department of otolaryngology,
Okayama University Hospital after acquisition of informed
consent from each patient. Submandibular gland or muscle far
and free from tumor invasion was obtained as normal control in
our experiments. All tissues were frozen in liquid nitrogen
immediately after surgery and stored $-80^{\circ} \mathrm{C}$ until the extraction
of DNA and RNA. Histological studies were also performed at
the department of pathology, and all tumors were confirmed as
squamous cell carcinoma.

DNA and RNA extractions
DNA and RNA extractions were performed as described in detail elsewhere ${ }^{2,3)}$.

\section{Microsatellite analysis}

Primers for amplification of microsatellite markers D12S372, D12S825, D12S77, D12S1697, D12S89 and D12S391 are available through the internet genome database (http:// www.gdb.org). PCR amplification and silver staining were performed as described previously ${ }^{2,3)}$.

Real time quantitative reverse transcriptase-polymerase chain reaction ( $R T-P C R)$

Real time quantitative RT-PCR was performed as described previously ${ }^{2,3)}$. The value of tumor specific ING4 expression was determined by calculating the ratio of the expression level in the tumor and that in the matched normal sample, each of which was normalized for the corresponding Cyclophilin expression level (T: ING4/Cyclophilin expression ratio in tumor sample; N: ING4/ Cyclophilin expression ratio in matched normal sample; $\mathrm{T} / \mathrm{N}$ ratio was the relative ING4 expression in tumor sample). Decreased and increased expression levels were defined when this ratio was $\leq 0.75$ ( $25 \%$ or more decrease) and $\geq 1.25$ ( $25 \%$ or more increase), respectively. Reproducibility was confirmed by independent PCR repeated twice.

\section{Mutation analysis of ING4}

Mutation analysis was performed by RT-PCR and direct sequencing as described previously ${ }^{2,3)}$.

\section{Results}

Mapping of chromosome 12p12-13 region

By using homology search in genome database, we noticed the existence of a new member of ING family, ING4 gene at 12p13.31, in which frequent deletion has been reported in various cancers. To investigate the role of ING4 in head and neck cancers, we first redefined the mapping of 12p12-13 region by searching genome database and using contiguous sequences (http: // 
J.Hard Tissue Biology.14(2)Proceeding,2005

Table 1

Analysis of LOH at Ch12p12 and ING4 mRNA expression

\begin{tabular}{|c|c|c|c|c|c|c|c|}
\hline Case No & Ratio & Class & $\mathrm{LOH}$ & Case No & Ratio & Class & $\mathrm{LOH}$ \\
\hline 1 & 0.41 & $\mathrm{~L}$ & + & 29 & 4 & $\mathrm{H}$ & + \\
\hline 2 & 0.5 & $\mathrm{~L}$ & - & 30 & 0.41 & $\mathrm{~L}$ & + \\
\hline 3 & 0.45 & $\mathrm{~L}$ & + & 31 & 1.2 & $\mathrm{~N}$ & + \\
\hline 4 & 0.1 & $\mathrm{~L}$ & - & 32 & 1.41 & $\mathrm{H}$ & + \\
\hline 5 & 0.76 & $\mathrm{~N}$ & + & 33 & 2.76 & $\mathrm{H}$ & + \\
\hline 6 & 0.49 & $\mathrm{~L}$ & + & 34 & 0.18 & $\mathrm{~L}$ & + \\
\hline 7 & 0.31 & $\mathrm{~L}$ & - & 35 & 0.25 & $\mathrm{~L}$ & + \\
\hline 8 & 0.1 & $\mathrm{~L}$ & + & 36 & 2.58 & $\mathrm{H}$ & - \\
\hline 9 & 0.61 & $\mathrm{~L}$ & + & 37 & 0.26 & $\mathrm{~L}$ & - \\
\hline 10 & 0.7 & $\mathrm{~L}$ & - & 38 & 0.41 & $\mathrm{~L}$ & + \\
\hline 11 & 0.24 & $\mathrm{~L}$ & - & 39 & 0.29 & $\mathrm{~L}$ & - \\
\hline 12 & 2 & $\mathrm{H}$ & + & 40 & 0.35 & $\mathrm{~L}$ & + \\
\hline 13 & 0.16 & $\mathrm{~L}$ & - & 41 & 0.32 & $\mathrm{~L}$ & + \\
\hline 14 & 0.1 & $\mathrm{~L}$ & + & 42 & 0.1 & $\mathrm{~L}$ & + \\
\hline 15 & 0.18 & $\mathrm{~L}$ & + & 43 & 0.39 & $\mathrm{~L}$ & + \\
\hline 16 & 1.02 & $\mathrm{~N}$ & + & 44 & 0.46 & $\mathrm{~L}$ & + \\
\hline 17 & 0.29 & $\mathrm{~L}$ & + & 45 & 0.7 & $\mathrm{~L}$ & - \\
\hline 18 & 0.14 & $\mathrm{~L}$ & + & 46 & 0.2 & $\mathrm{~L}$ & - \\
\hline 19 & 0.53 & $\mathrm{~L}$ & + & 47 & 0.17 & $\mathrm{~L}$ & - \\
\hline 20 & 1.04 & $\mathrm{~N}$ & + & 48 & 0.14 & $\mathrm{~L}$ & + \\
\hline 21 & 0.27 & $\mathrm{~L}$ & + & 49 & 0.42 & $\mathrm{~L}$ & - \\
\hline 22 & 0.46 & $\mathrm{~L}$ & + & 50 & 0.36 & $\mathrm{~L}$ & + \\
\hline 23 & 2.4 & $\mathrm{H}$ & - & \multirow{3}{*}{\multicolumn{4}{|c|}{$\begin{array}{l}\text { The ratio of the expression levels in the matched tumor } \\
\text { and normal samples was calculated as described in } \\
\text { materials and methods. Decreased and increased expression }\end{array}$}} \\
\hline 24 & 0.55 & $\mathrm{~L}$ & + & & & & \\
\hline 25 & 0.85 & $\mathrm{~N}$ & + & & & & \\
\hline 26 & 0.17 & $\mathrm{~L}$ & - & \multicolumn{4}{|c|}{ levels were shown as classes $\mathrm{L}$ and $\mathrm{H}$ when this ratio was d" } \\
\hline 27 & 0.36 & $\mathrm{~L}$ & - & \multicolumn{4}{|c|}{0.75 and e” 1.25 , respectively. Class $\mathrm{N}$ means the } \\
\hline 28 & 2.38 & $\mathrm{H}$ & - & \multicolumn{4}{|c|}{ value of ratio between 0.75 and 1.25 . } \\
\hline
\end{tabular}

www.ncbi.nlm.nih.gov/genome/guide/human/, http:// www.gdb.org/).

Loss of heterozygosity analysis of chromosome 12p12-13 region We selected 6 microsatellites markers on chromosome 12p1213 to examine allelic loss in ING4 location. We analyzed DNAs from 50 pairs of matched HNSCC and normal tissues for losses at microsatellite markers D12S372, D12S825, D12S77, D12S1697, D12S89, and D12S391. Overall, LOH was detected at least one location in 33 of 50 (66\%) samples. The most frequent loss (66\%) was seen at marker D12S825, which is about 700 bps away from ING4 location. All these LOH data pointed out ING4 location as a hot target area in HNSCC.

\section{Mutation analysis of ING4 gene}

We examined all coding region of ING4 for mutation at RNA level by RT-PCR and direct sequencing in 50 HNSCC. However no somatic change was detected in any of the tumor samples.

ING4 mRNA expression analysis

We examined expression level of ING4 mRNA in 50 tumor samples comparing with the paired normal tissues by quantitative real-time RT-PCR analysis to find other possible inactivation mechanisms. Thirty-eight out of 50 (76\%) tumor tissues showed decreased expression as compared with that of each paired normal tissue, while only 5 of 50 (10\%) samples demonstrated similar level of the expression in normal and tumor tissues. Most of those samples with low expression demonstrated over $70 \%$ decrease in ING4 expression in tumors as compared to their normal counterparts. In $14 \%$ (7/50) of samples, increased expression of ING4 was detected in tumor tissues (Table 1).

\section{Discussion}

Our studies involving ING1 and ING3 suggest that ING family is an important group of genes in the carcinogenic pathway of at least head and neck cancers. Recent progress in human sequencing displayed two new members of this family ING4 and ING5.

We did not detect any somatic mutation of ING4. Mutation analysis was performed at RNA level using cDNAs prepared from tumor samples and therefore mutant RNAs may not be stable and could be easily destroyed. However, decreased expression of ING4 was detected $76 \%$ of tumor samples as compared to their normal counterparts with quantitative real-time PCR. Most of those samples with low ING4 expression displayed extreme mRNA loss. 
International symposium of Maxillofacial \& Oral Regenerative Biology in Okayama 2005

Rather epigenetic inactivation or abnormalities of upstream genes could be a reason for decreased ING4 expression which may eventually result in haploinsufficiency of it. This haploinsufficiency, described for some genes including TGF $\beta 1$, INK4c, could make epithelial cells more susceptible to carcinogens like smoking which is very commonly seen in patients with HNSCC. Further studies both for different inactivation mechanisms of ING4 and its role in carcinogenesis of HNSCC are warranted. Recent studies and our studies suggested that ING4 might play an important role in various cancers including HNSCC. Nevertheless this region also includes some genes, of which functions are yet analyzed. Further studies of genes both already known and unknown should be performed to clarify their role in cellular processes.

\section{References}

1. Bai, F., Pei, X.H., Godfrey, V.L., Xiong, Y.: Haploinsufficiency of p18 (INK4c) sensitizes mice to carcinogen-induced tumorigenesis. Mol. Cell. Biol. 23, 1269-1277, 2003.

2. Gunduz, M., Ouchida, M., Fukushima, K., Hanafusa, H., Etani, T., Nishioka, S., Kazunori, N., Shimizu, K.: Genomic structure of the human ING1 gene and tumor-specific mutations detected in head and neck squamous cell carcinomas. Cancer Res. 60,3143-3146, 2000.

3. Gunduz, M., Ouchida, M., Fukushima, K., Ito, S., Jitsumori, Y., Nakashima, T., Nagai, N., Nishizaki, K., Shimizu, K.: Allelic loss and reduced expression of the ING3, a candidate tumor suppressor gene at $7 q 31$, in human head and neck cancers. Oncogene 21, 4462-4470, 2002. 